\title{
Exploiting Addresses Correlation to Maximize Lifetime of IPv6 Cluster-based WSNs
}

\author{
Leila Ben Saad ${ }^{* \ddagger}$, Bernard Tourancheau ${ }^{\dagger \ddagger}$ \\ * ENS de Lyon, INRIA \\ $\dagger$ Université de Lyon 1, INRIA \\ $\ddagger$ INSA-Lyon, CITI, F-69621, Villeurbanne \\ leila.ben.saad@ens-lyon.fr, Bernard.Tourancheau@INRIA.fr
}

\begin{abstract}
Improving the network lifetime is an important design criterion for wireless sensor networks. To achieve this goal, we propose in this paper a novel approach which applies source-coding on addresses in heterogeneous IPv6 Cluster-based wireless sensor network. We formulate the problem of maximizing the network lifetime when Slepian-wolf coding is applied on addresses in network composed of line-powered and batterypowered sensors. This problem optimizes the placement of linepowered sensors to enable the battery-powered ones to exploit the addresses correlation and reduce the size of their emitted packets and thus improve the network lifetime. The numerical results show that a significant network lifetime improvement can be achieved (about $25 \%$ in typical scenario).
\end{abstract}

Index Terms-Network lifetime, Slepian-Wolf source-coding, addresses, heterogeneous IPv6 Cluster-based WSNs.

\section{INTRODUCTION}

Due to the recent advances achieved in wireless communications and microelectronics, the use of wireless sensor networks (WSNs) has witnessed a considerable increase in a wide range of applications such as in home and building monitoring. Such networks are composed of thousands of battery-powered sensors with limited processing and memory capabilities. These sensors have to operate autonomously, monitor their environment and regularly report the collected measurements (e.g. temperature, humidity, light) towards the sink. To fulfill the requirements of such applications, the sensors are expected to operate for several years without replacing the batteries. Therefore, improving the network lifetime is an important design criterion in WSNs. To achieve this goal, the sensors' energy must be saved.

The energy spent in the radio communication represents the most consuming part in the sensor activity. That is why, minimizing the number and the size of the packets transmitted by the sensors by means of data compression or aggregation, has been considered as an important mean of improving WSN lifetime and has been adopted in many works [1]. Moreover, organizing the sensors in clusters to increase the network lifetime has been widely pursued by several studies [2]. In addition, using energy heterogeneity in WSNs by the introduction of nodes with enhanced energy capacity has also been shown as an efficient way to sustain a long lifetime of the WSNs in many researches [3].

This work combines the above approaches i.e. clustering, heterogeneity and compression to increase the network life- time of WSNs. The network is divided into several clusters. Each cluster contains two types of sensors: batterypowered and line-powered. The number of bits transmitted by the battery-powered sensors towards the sink is reduced by compression. However, contrarily to the majority of the previous works where the compression is applied on data i.e payload [1], this work deals with the compression of the control part of the packet which consists roughly in the addresses especially with IPv6. The size of the packets emitted by the battery-powered sensors is reduced by leveraging the correlation between the addresses. The main idea is that the battery-powered sensors use source-coding to encode in most concise way their addresses based on the overheard addresses of line-powered ones.

In this paper in order to increase the network lifetime, we propose to apply Slepian-Wolf coding on addresses in heterogeneous IPv6 Cluster-based WSN. We also formulate the problem of network lifetime maximization when this approach is used. The optimization of the placement of linepowered sensors is conducted to enable the battery-powered ones to exploit the addresses correlation and reduce the size of their emitted packets and thus improve the network lifetime.

The remainder of this paper is organized as follows. Section 2 reviews the main techniques proposed in the literature to improve the network lifetime. In Section 3, the major assumptions as well as the network and the power consumption models are described. Section 4 studies how to apply SlepianWolf source-coding on addresses to improve the network lifetime. Section 5 proposes a formulation of the problem of network lifetime maximization when source-coding on addresses is applied. Section 6 evaluates the performance of the proposed approach and presents the numerical results. Section 7 concludes the paper.

\section{RELATED WORK}

More and more efforts have been done recently to improve the lifetime of WSNs. Indeed, there has been an increasing interest in energy harvesting to sustain the operational lifetime of sensors [4]. Moreover, several energy-efficient networking protocols aimed to conserve sensors energy have been proposed e.g. topology control [5], routing [6] and clustering [2]. Many others techniques have been proposed to reduce the energy consumption [7] e.g. duty cycling, power management, 
cross-layering. Another technique, recently used as a way to increase the network lifetime is the sinks mobility [8][9][10]. One of the most promising approach for network lifetime improvement is data compression or aggregation which aims to minimize the amount of data to be transmitted by the sensors [1][11]. In this context, some works as in [12][13][14][15] proposed to use distributed source-coding in cluster-based WSN. The objective of these works was to remove the redundancy observed in sensed data due to the spatio-temporal correlation. However, most of these works deals with data compression (i.e. payload) without considering the addresses compression. While at the same time, the size of the control part in the emitted packet is often higher than the payload in WSNs which leads to non negligible energy consumption.

There are two studies closely related to our work in the sense that they deal with the compression of the control part. The first one is known as header compression [16]. The key idea of this technique comes from the observation that correlation exists between consecutive packets of the same flow: some fields remain static or can be inferred from others. The header compression method reduces the size of the header by eliminating the redundant fields and transmitting only the fields carrying information. A common approach in header compression is first to transmit a packet with an uncompressed header and then transmit only the differences between the current packet and previous one. A packet with an uncompressed header defines the context in a flow. It is associated with a context identifier which is known at the compressor and the decompresser. With respect to this context the packet is decompressed at the receiver side.

Some standards based on header compression technique were proposed by the IETF, e.g. IP Header Compression IPHC [17], RObust Header Compression ROHC [18]. IPHC is suitable for low bit error rate links whereas ROHC is suitable for high bit error rate links and long round trip time. These standards can be performed only over a link between two nodes. To tackle this shortcoming and allow header compression in 6LoWPAN networks, others header compression mechanisms were proposed by the IETF e.g. LOWPAN_HC1 [19], LOWPAN_HC1g [20] and LOWPAN_IPHC [21]. In LOWPAN_HC1 and LOWPAN_HClg, the size of the header is reduced without the use of any context by eliminating the fields that are repeated through all the flows or that can be inferred from lower layers. The disadvantage of LOWPANHC1 is that the full 128 bits address must be carried in line in multicast and global communication. While, the drawback of LOWPANHC1g is that the full 128 bits address must be used when sensors communicate with external 6LowPAN networks. To improve these techniques, LOWPAN_IPHC [21] was proposed. It is context-based compression and it relies on information related to the entire 6LoWPAN network. With this method, the 128-bits routable addresses can be reduced to two bytes when routing over multiple IP hops. To sum up, 6LoWPAN header compression techniques have significantly reduced the control part of the packets. However, to supervise, control and monitor WSNs applications from outside of the network, routable addresses should be used. Despite the fact that significant reduction has been achieved on such addresses, there is still room of improvement.

The second work related to the compression of the control part was proposed by Kronewitter in [22]. It is based on Huffman codes. Each sensor has a weight which is the inverse of its residual energy. This weight is used to build an Huffman tree which is used to define the addresses. Therefore, short addresses are assigned to the sensors with the lowest energy level. A shortcoming of this approach is that it is centralized: a gateway need to know the energy map of the sensors. Moreover, many messages need to be exchanged in order to update the Huffman tree and the addresses.

\section{MAJOR ASSUMPTIONS}

In this section, the major assumptions as well as the network and the power consumption models are described.

\section{A. Network Model}

In the applications dedicated to home and buildings surveillance, WSNs are often composed of many battery-powered sensors, some line-powered sensors and sinks. In this work, we assume that the network is divided into medium-sized clusters. Each cluster contains only one sink which collects regularly the sensed measurements from battery-powered sensors and line-powered ones. Since the line-powered sensors must be plugged to a wall outlet, it is assumed that their number is very small and that they can be placed only at a subset of candidate locations within the boundary of the cluster (see Fig 1).

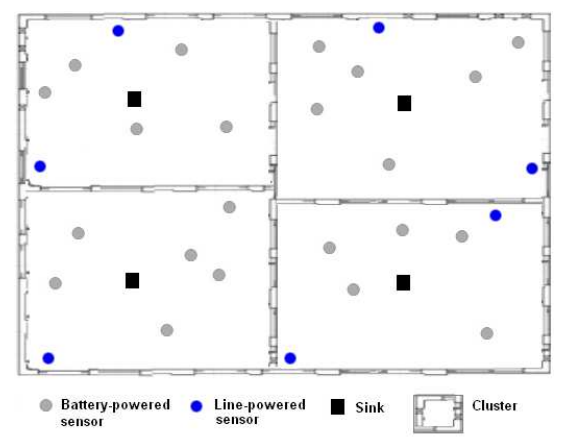

Fig. 1. Battery-powerd sensors, line-powered sensors and sinks inside a building

The battery-powered sensors are randomly distributed in the cluster and have the same amount of energy capacity. A timedriven sampling application of data collection is considered where each sensor regularly generates the same amount of data. It is assumed that a direct transmission is used by the sensors to send their packets to the sink. This can be justified by the fact that the direct transmission is more energy efficient than multi-hop routing in small-to-medium sized WSN [12]. Each sensor is supposed to use omni-directional antenna. For sake of simplicity, it is assumed that noise free communications occur between the sensors. In this work, we are interested 
in the source addresses of the emitted packets. The addresses allocated to the battery-powered sensors and the line-powered ones are assumed to be highly correlated. This means that the source addresses of the packets emitted by the batterypowered sensors and the line-powered ones differ only in one bit. This can be guaranteed by an appropriate algorithm of addresses allocation. It is also assumed that each line-powered sensor is scheduled to usually transmit its packet before the battery-powered one. This enables the latter ones to exploit the existing correlation between the addresses. This can be obtained by an adequate protocol where the battery-powered sensors are weak-up by the line-powered ones. There are many network lifetime definitions which depend on applications. In this paper, the widely used one is adopted where the network lifetime is defined as the time until the first battery-powered sensor dies i.e. it uses up all its residual energy.

In this work, we focus to improve the WSN lifetime by reducing the size of the transmitted packets by applying Slepian-Wolf source-coding on addresses. We also seek to address this question:

What is the network lifetime gain that can be achieved if the battery-powered sensors reduce the size of their own addresses based on the overheard addresses of line-powered ones which are optimally placed?

\section{B. Power Consumption Model}

To calculate the power consumption in the WSN, the same realistic model used in [23][24] is considered. Therefore, the power expended in $\mathrm{J} / \mathrm{s}$ to receive $b_{1}-\mathrm{bit} / \mathrm{s}$ in the same radio model is:

$$
P_{R x}\left(b_{1}\right)=b_{1} \times E_{e}
$$

where $E_{e}(\mathrm{~J} / \mathrm{bit})$ is the energy spent per bit in the electronics circuits at the transceiver.

The power expended in $\mathrm{J} / \mathrm{s}$ to transmit $b_{2}$-bit/s to a distance $d$ is:

$$
\left.P_{T x}\left(b_{2}, d\right)\right)=b_{2} \times\left(E_{e}+E_{a m p} \times d^{\beta}\right)
$$

where $E_{a m p} \times d^{\beta}(\mathrm{J} / \mathrm{bit})$ is the energy consumed per bit at the amplifier. $d$ is the distance between the transmitting and the receiving sensor. $\beta$ is the path loss exponent.

$E_{e}+E_{a m p} \times d^{\beta}(\mathrm{J} / \mathrm{bit})$ is the total energy consumption when transmitting one bit over a distance of $d$.

The power consumed in $\mathrm{J} / \mathrm{s}$ to compress $b_{3}$-bit/s is:

$$
P_{C p}\left(b_{3}\right)=b_{3} \times E_{c}
$$

where $E_{c}(\mathrm{~J} / \mathrm{bit})$ is the energy consumed by the processor to process one bit of information in the packet during compression. Notice that a simple model for energy spent in compression is assumed. In general, $E_{c}$ ranges from $5 \mathrm{~nJ} /$ bit to $50 \mathrm{~nJ} / \mathrm{bit}[12][15][24]$.

The energy spent by the sensor when it is idle is assumed negligible (i.e. ideal scenario is assumed where the transceiver is turn off when not used).

\section{SOURCE-CODING ON ADDRESSES}

In order to reduce the size of the transmitted addresses, the battery-powered sensors exploit the overhearing of the addresses of line-powered sensors by applying the Slepianwolf coding method [25]. The potential benefit of using this well-known method is that it offers a high compression rate in high correlated data.

\section{A. Slepian-wolf coding}

Slepian-Wolf coding [25] is a lossless distributed sourcecoding technique that eliminates the redundancy observed in correlated discrete sources in order to encode them in the most concise way. The Slepian-wolf theorem [25] states that two random correlated sources $\mathrm{X}$ and $\mathrm{Y}$, encoded independently, and then decoded jointly at a receiver, can be lossless encoded at a rate lower bounded by their joint entropy $H$, even without any communication between the two sources.

$$
H(X, Y) \leq H(X)+H(Y)
$$

The results of Slepian and Wolf also show that it is possible to encode them at a rate equal to their joint entropy:

$$
H(X, Y)=H(X)+H(Y \mid X)
$$

Practical techniques based on Slepian-Wolf coding have been found recently (see [26] for instance). In this work, in order to achieve the bounds defined by Slepian and Wolf and reduce the size of the emitted packets, syndrome coding [27] is applied on the addresses as described in our following work [28].

\section{B. Application of syndrome coding on addresses}

Let consider a simple network composed of a line-powered sensor $S_{1}$ and a battery-powered sensor $S_{2}$ having respectively the source addresses $A_{1}$ and $A_{2}$ (see Fig 2). The correlation between these two addresses is very high.

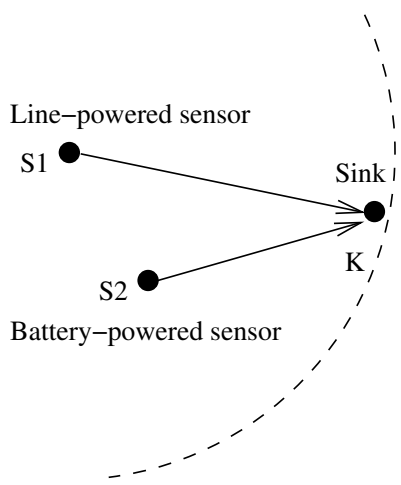

Fig. 2. Battery-powered sensor $S_{2}$ overhearing the packet of line-powered sensor $S_{1}$ destinated to the sink $K, d_{S_{1} S_{2}}<d_{S_{1} K}$

The line-powered sensor $S_{1}$ send a packet to the sink $K$. The battery-powered sensor $S_{2}$ overhears this packet and decides to exploit this overhearing by sending to the sink a packet with address length (in bits) $H\left(A_{2} \mid A_{1}\right)$ instead of 
$H\left(A_{2}\right)$. To reach this address length, the battery-powered sensor $S_{2}$ chooses an $[n, k, d]$ linear code $\mathcal{C}$ over the finite field $\mathbb{F}_{2}$ where $n$ is the code length, $k$ is the code dimension and $d$ is the minimum weight of the code. The code length $n$ is equal to size of the addresses $A_{1}$ and $A_{2}$ which are viewed as vectors over $\mathbb{F}_{2}$ of length $n$. The code $\mathcal{C}$ must be chosen such that $A_{1}$ or $A_{2}$ is a codeword of $\mathcal{C}$. Let assume that $A_{1}$ is a codeword of $\mathcal{C}$. Instead of sending $A_{2}$, the sensor $S_{2}$ can transmit the syndrome of $A_{1} \oplus A_{2}$ for $\mathcal{C}$ :

$$
\sigma\left(A_{1} \oplus A_{2}\right)=\left(A_{1} \oplus A_{2}\right) \cdot \mathcal{H}^{t}
$$

With $\mathcal{H}$ the parity check matrix of this code $\mathcal{C}$.

The syndrome of $A_{1} \oplus A_{2}$ sent by $S_{2}$ as address is viewed as an "error". The sink can recover this error from the knowledge of $A_{1}$ and $\sigma\left(A_{1} \oplus A_{2}\right)$ by using standard arrays for instance (see [29]). This method succeeds if and only if $d$ is the smallest possible minimum weight for a binary linear code that is greater or equal to $2 d_{H}\left(A_{1}, A_{2}\right)+1$ with $d_{H}$ the Hamming distance function. The dimension of the syndrome $\sigma: n-k$ represents the compression gain of this method.

If $d_{H}\left(A_{1}, A_{2}\right)=1$ and the size the address allocated to the sensors is $n=16$, the sensor $S_{2}$ can use the $[16,11,4]$ code to send a 5 -bits address to the sink. If $n=32, S_{2}$ can use the $[32,26,4]$ code to send a 6-bits address to the sink. If $n=64$, $S_{2}$ can use the $[64,57,4]$ code to send a 7-bits address to the sink. If $n=128, S_{2}$ can use the $[128,120,4]$ code to send a 8-bits address to the sink.

This shows that with Slepian-wolf coding method it is possible to reduce 128-bits address to 1 byte. It is better than LOWPAN_IPHC [21] where it can be reduced only to 2 bytes.

\section{Condition of address compression efficiency}

Let $U$ and $X$ denote respectively the uncompressed size of the packet emitted by the sensor and the uncompressed length of its source address. Let $u$ and $x$ denote respectively the size of the compressed packet and the length of the compressed source address used when source-coding is applied. The compression ratio denoted here $\gamma$ is:

$$
\gamma=\frac{u}{U}=\frac{U-(X-x)}{U}
$$

Let assume that the sensors $S_{1}$ and $S_{2}$ transmit their packets to the sink with the same rate $b_{1}=b_{2}=b(\mathrm{bit} / \mathrm{s})$. The power consumed by the sensor $S_{2}$ to transmit a packet to the sink over the distance $d_{S_{2} K}$ without addresses compression is:

$$
P_{S_{2}}=b \times\left(E_{e}+E_{a m p} \times d_{S_{2} K}^{\beta}\right)
$$

The power consumed by the sensor $S_{2}$ when its address is compressed based on the address of $S_{1}$ is equal to the power consumed in receiving from $S_{1}$, compressing and transmitting to the sink:

$$
P_{S_{2} / S_{1}}=b \times E_{e}+b \times E_{c}+\gamma \times b \times\left(E_{e}+E_{a m p} \times d_{S_{2} k}^{\beta}\right)
$$

In order to make the address compression beneficial for the sensor $S_{2}$. We must have:

$$
P_{S_{2} / S_{1}}<P_{S_{2}}
$$

This implicates that:

$$
d_{S_{2} K}>\left(\frac{E_{e} \times \gamma+E_{c}}{E_{a m p} \times(1-\gamma)}\right)^{\frac{1}{\beta}}
$$

Let $d_{\min }$ denotes the minimum distance bound for which it is beneficial for a sensor to use Slepian-wolf source-coding.

$$
d_{\text {min }}=\left(\frac{E_{e} \times \gamma+E_{c}}{E_{a m p} \times(1-\gamma)}\right)^{\frac{1}{\beta}}
$$

When the distance between a battery-powered sensor and the sink is less that $d_{\text {min }}$, it is ineffective to use source-coding on addresses because in this case the sensor will consume more energy in comparison to the case of no address compression is used.

\section{NeTwork LifETIMe Formulation}

In this section, the network lifetime optimization problem in cluster-based WSN is formulated. The network is partitioned in several medium-sized clusters (see Fig. 1). Each cluster contains one sink, line-powered sensors and battery-powered sensors. The network in each cluster can be represented by the graph $G(\mathcal{V}, \mathcal{E})$, where $\mathcal{V}$ denotes the set of all nodes in the cluster, $\mathcal{V}=\mathcal{S} \cup \mathcal{Y} \cup\{k\}$ and $\mathcal{E}$ denotes the set of wireless links, $\mathcal{E} \subseteq \mathcal{V} \times \mathcal{V}$.

- $\mathcal{S}$ denotes the set of battery-powered sensors.

- $\mathcal{Y}$ denotes the set of line-powered sensors.

- $k$ is the sink node.

- $\mathcal{F}$ denotes the set of all possible locations of line-powered nodes in the cluster, $|\mathcal{Y}|<|\mathcal{F}|$.

- There are $\left(\begin{array}{c}|\mathcal{F}| \\ |\mathcal{Y}|\end{array}\right)$ configurations to place the $|\mathcal{Y}|$ poweredline nodes among $|\mathcal{F}|$ locations. Let $\mathcal{L}$ denotes the set of all these possible configurations.

- $e_{0}(\mathrm{~J})$ is the initial energy of each battery-powered sensor.

- $r_{i k}^{l}(\mathrm{bit} / \mathrm{s})$ is the data transmission rate from node $i$ to the sink $k$ where the configuration $l \in \mathcal{L}$ is used, $i \in \mathcal{S}$.

- $g_{r}(\mathrm{bit} / \mathrm{s})$ is the rate at which data packets are generated.

- $d_{i j}$ denotes the distance between node $i$ and $j, i, j \in \mathcal{V}$, $(i, j) \in \mathcal{E}$

- $\mathcal{N}_{i}^{l}$ is the set of line-powered nodes placed according to the configuration $l$ and their packets transmission to the sink can be heard by the battery-powered sensor $i$. $\mathcal{N}_{i}^{l}=\left\{j \in \mathcal{Y} \mid d_{j i} \leq d_{j k}\right\}, i \in \mathcal{S}, l \in \mathcal{L}$.

- $\delta_{i}$ is a binary variable indicating if the source-coding on addresses is applied or not.

- $p_{i}^{l}(\mathrm{~J} / \mathrm{s})$ is the power consumed by the battery-powered sensor $i$ where the line-powered sensors are placed according to the configuration $l, l \in \mathcal{L}$.

- $t_{i}^{l}$ is the lifetime of the $i$ th battery-powered sensor where the line-powered sensors are placed according to the configuration $l, l \in \mathcal{L}$.

- $\mathrm{T}$ is the network lifetime 
The network lifetime and the optimal placement of linepowered sensors are determined by solving the following maxmin optimization problem.

$$
T=\max _{l \in \mathcal{L}} \min _{i \in \mathcal{S}}\left\{t_{i}^{l}\right\},
$$

The lifetime $t_{i}^{l}$ is calculated as follows:

$$
t_{i}^{l}=\left(\frac{e 0}{p_{i}^{l}}\right), l \in \mathcal{L}, i \in \mathcal{S}
$$

The power $p_{i}^{l}$ is given by:

$$
\begin{array}{r}
p_{i}^{l}=\delta_{i} \times\left(g_{r} \times E_{e}+g_{r} \times E_{c}\right)+r_{i k}^{l} \times\left(E_{e}+E_{a m p} \times d_{i k}^{\beta}\right), \\
i \in \mathcal{S}, j \in \mathcal{N}_{i}^{l}, l \in \mathcal{L} \quad(12)
\end{array}
$$

$\delta_{i}$ and $r_{i k}^{l}$ depend on the configuration of the line-powered sensors and the distance between the battery-powered sensors and the sink. Their values are determined as follows:

$$
\forall i \in \mathcal{S}, l \in \mathcal{L}, \quad \delta_{i}= \begin{cases}1 & \text { if } \mathcal{N}_{i}^{l} \neq \varnothing \text { and } d_{i k}>d_{\text {min }} \\ 0 & \text { otherwise }\end{cases}
$$

$\forall i \in \mathcal{S}, l \in \mathcal{L}, \quad r_{i k}^{l}= \begin{cases}\gamma \times g_{r} & \text { if } \mathcal{N}_{i}^{l} \neq \varnothing \text { and } d_{i k}>d_{\text {min }} \\ g_{r} & \text { otherwise }\end{cases}$

This means that if a battery-powered sensor overhears a correlated address of a line-powered one and its distance to reach the sink is higher than $d_{m i n}$, it can apply source-coding to reduce its own address. Otherwise, no address compression is conducted.

\section{NUMERICAL RESUlTS}

To evaluate the performance of the proposed approach on network lifetime, numerical experiments were performed. The parameter specifications used in this study are summarized in Table I. Typical values of energy consumption are used as in [24][30].

TABLE I

PARAMETERS VALUES

\begin{tabular}{|l|l|}
\hline Parameter & value \\
\hline \hline$|\mathcal{S}|$ & 40 \\
$|\mathcal{Y}|$ & 4 \\
Packet size $U$ & $508,762,1016 \mathrm{bits}$ \\
Address size $X$ & $16,32,64,128 \mathrm{bits}$ \\
$g_{r}$ & 1 packet per hour \\
$E_{c}$ & $5,10,50 \mathrm{~nJ} / \mathrm{bit}$ \\
$E_{e}$ & $10,50,100 \mathrm{~nJ} / \mathrm{bit}$ \\
$\beta$ & 2 (free space large room) \\
$E_{a m p}$ & $100 \mathrm{pJ} / \mathrm{bit} / \mathrm{m}^{2}$ \\
$e 0$ & $16200 \mathrm{~J}(\mathrm{AA}$ battery, 3 Ahr, 1.5V) \\
Confidence interval & $95 \%$ \\
\hline
\end{tabular}

The battery-powered sensors are randomly distributed in square clusters. The sink is located at the center of each cluster. The line-powered sensors can be placed only at subset locations at the perimeter. It is assumed that their number per cluster is four. Their optimal locations are determined by

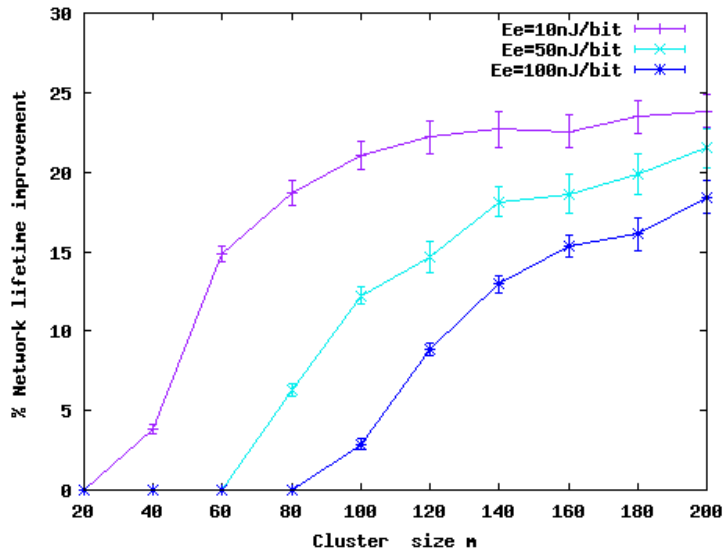

(a) Different values of $E_{e}$ by fixing $E_{c}$ to $5 \mathrm{~nJ} / \mathrm{bit}$

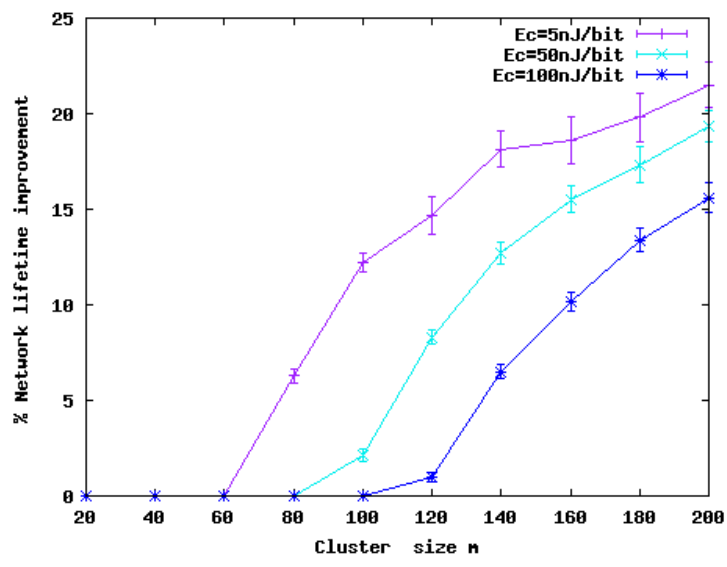

(b) Different values of $E_{c}$ by fixing $E_{e}$ to $50 \mathrm{~nJ} / \mathrm{bit}$

Fig. 3. Network lifetime improvement when source-coding on addresses is applied as function of the cluster size for different values of energy consumption. The following parameter values are used: $|\mathcal{S}|=40,|\mathcal{Y}|=4, \mathrm{U}=508$ bits, $X=128$ bits,$E_{e}$ in $\{10,50,100\} \mathrm{nJ} / \mathrm{bit}, E_{c}$ in $\{5,50,100\} \mathrm{nJ} / \mathrm{bit}$

the resolution of the max-min optimization problem of the equation (10).

We investigated the impact of the cluster size, the energy consumption, the packet and the address size on network lifetime improvement, when source-coding on addresses is applied with respect to the case of no address compression. The network lifetime improvement is obtained by conducting 200 experiments and by averaging the results. Each experiment uses a different randomly generated network.

Figures 3 and 4 show that the cluster size affects considerably the network lifetime improvement. In fact, when the cluster size increases, the lifetime gain becomes more important. This can be explained by the fact that when the average distance between the sensors and the sink increases, the sensors may have more opportunity to compress their addresses and thus conserve energy when sending packets to the sink. As it has already been shown in section IV, the source-coding on addresses can be beneficial only when this distance is greater than the minimum distance bound $d_{m i n}$.

In Figures 3(a) and 3(b), we evaluate the impact of energy 


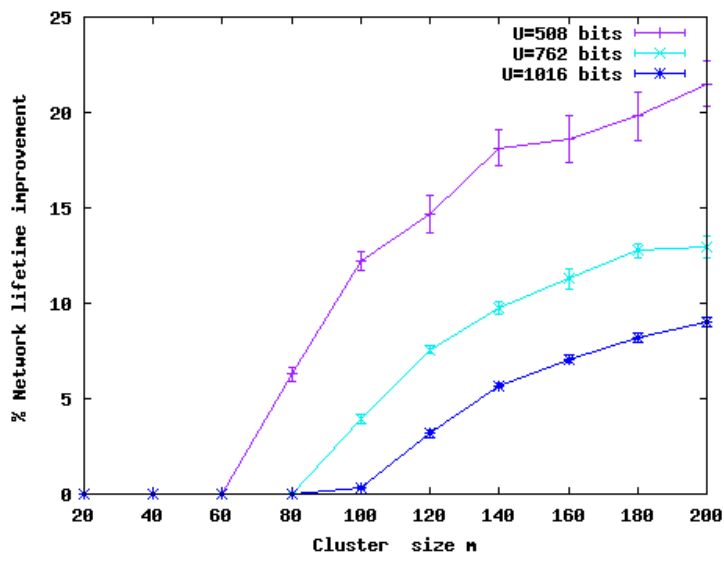

(a) Different packet sizes $U$ bits, $X=128$ bits

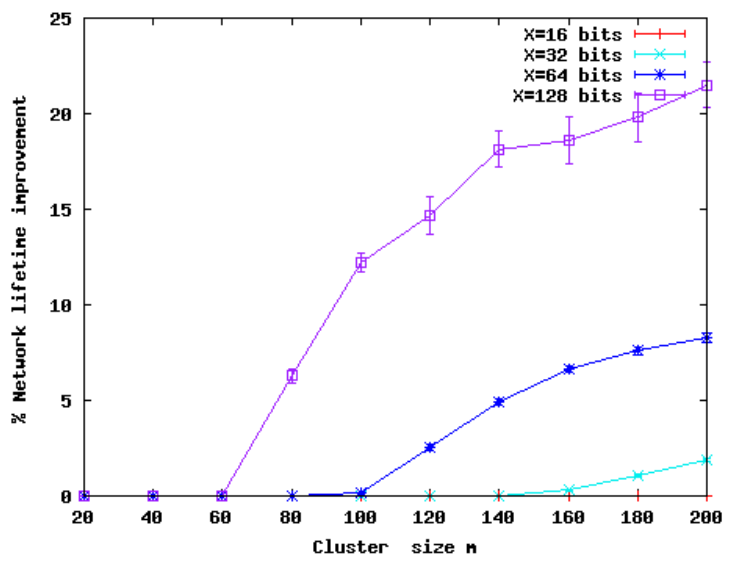

(b) Different address sizes $X$ bits, $U=508$ bits

Fig. 4. Network lifetime improvement when source-coding on addresses is applied as function of the cluster size for different addresses and packets sizes. The following parameter values are used: $|\mathcal{S}|=40,|\mathcal{Y}|=4, E_{e}=50 \mathrm{~nJ} / \mathrm{bit}$ $E_{c}=5 \mathrm{~nJ} / \mathrm{bit}$

spent respectively in electronics circuits at the transceiver and in compression at the processor on network lifetime gain. As expected, the lifetime improvement decreases when $E_{e}$ or $E_{c}$ grows. Nevertheless even with high values of energy consumption, about $15 \%$ of lifetime improvement can be achieved with source-coding on 128 bits addresses.

Figure 4 analyses the network lifetime gain for different packets and addresses sizes. As shown in the Figure 4(a), the packets' sizes used are 508, 762, 1016 bits (i.e. MTU 802.15.4). Notice that in WSN applications, the size of the packets is often small to avoid the increase of error rates. The compression ratios $\gamma$ obtained with these packets' sizes when applying source-coding on addresses are respectively 0.76 , 0.84 and 0.88 . Despite the fact that the gain decreases when the packet size gets longer due to the increase of compression ratio, the lifetime improvement is still about $9 \%$ for a packet of size 1016 bits. In Figure 4(b), the addresses' sizes used are 16 bits (i.e. IEEE 802.15.4 short address), 32 bits (i.e. IPv4 address), 64 bits (i.e. IEEE 802.15 .4 extended address) and 128 bits (i.e. full IPv6 address). The compression ratios $\gamma$ obtained

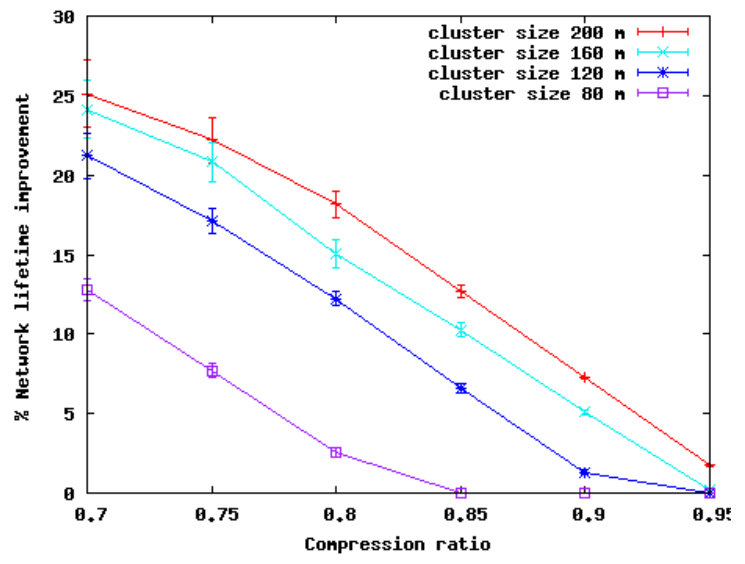

Fig. 5. Network lifetime improvement when source-coding on addresses is applied as function of compression ratio for different cluster size. The following parameter values are used: $|\mathcal{S}|=40,|\mathcal{Y}|=4, E_{e}=50 \mathrm{~nJ} / \mathrm{bit}, E_{c}=5 \mathrm{~nJ} / \mathrm{bit}$

with source-coding on these addresses are respectively 0.76 , $0.88,0.94$ and 0.97 . As expected, the network lifetime gain decreases when the address size gets shorter due to the increase of compression ratio. When the address size is too small i.e. 16 bits, it is ineffective to apply source-coding because no gain in lifetime can be achieved.

Figure 5 shows that the network lifetime gain depends considerably on the compression ratio $\gamma$. Nevertheless, a significant network lifetime improvement can be achieved by applying source-coding on addresses in heterogeneous clusterbased WSNs. This improvement can range from 2.5\% to $25 \%$ in cluster size higher than $80 \mathrm{~m}$ with a compression ratio less than 0.8 .

To sum up, let consider a typical building network of $400 \times 400 \mathrm{~m}$. This network is divided in 4 clusters of $200 \times 200 \mathrm{~m}$. Each cluster is composed of 40 battery-powered sensors and 4 line-powered sensors optimally placed according to the solution of the max-min optimization problem. The sensors send a packet of 400-bits size with 128-bits address every one hour. By applying source-coding on addresses, the lifetime improvement that can be achieved reaches $25 \%$.

\section{CONCLUSiON}

In this paper, the application of Slepian-Wolf source-coding on addresses in heterogeneous IPv6 Cluster-based WSNs is proposed. The clustering problem was set as an optimization problem that maximizes the network lifetime when SlepianWolf coding is used. The placement of line-powered nodes is optimized to enable the battery-powered nodes to exploit the addresses correlation and reduce the size of their emitted packets and thus improve the network lifetime. With SlepianWolf coding, 128 bits addresses can be reduced to 1 byte. It is a significant improvement especially when routable addresses are needed in networks communicating outside of the 6LoWPAN networks. Numerical results show that the lifetime improvement that can be achieved with this approach reaches $25 \%$ in typical scenario. This approach can be combined with 
data compression to achieve further network lifetime improvement. In future works, we intend to study how to allocate the addresses in order to guarantee the optimal network lifetime and how to evaluate its performance in multi-hop large scale networks.

\section{ACKNOWLEDGEMENTS}

We greatly thank Cédric Lauradoux and Fabrice Valois for their helpful discussions about source coding on addresses that leaded to the optimization problem described in this paper.

\section{REFERENCES}

[1] R. Rajagopalan and P. K. Varshney, "Data aggregation techniques in sensor networks: A survey," IEEE Communications Surveys and Tutorials, vol. 8, pp. 48-63, 2006.

[2] A. A. Abbasi and M. Younis, "A survey on clustering algorithms for wireless sensor networks," Computer Communications, vol. 30, pp. 2826-2841, October 2007.

[3] M. Yarvis, A. Kushalnagar, H. Singh, Y. Liu, and S. Singh, "Exploiting heterogeneity in sensor networks," in Proceeding of the IEEE Infocom, 2005.

[4] R. Want, K. I. Farkas, and C. Narayanaswami, "Guest editors' introduction: Energy harvesting and conservation," IEEE Pervasive Computing, vol. 4, pp. 14-17, 2005.

[5] X. yang Li, W. zhan Song, and Y. Wang, "Topology control in heterogeneous wireless networks: Problems and solutions," in Proceedings of the 23 rd Joint Conference of the IEEE Computer and Communications Societies INFOCOM, 2004

[6] M. Perillo and W. Heinzelman, Wireless Sensor Network Protocols, fundamental algorithms and protocols for wireless and mobile networks ed. CRC Hall, 2005.

[7] G. Anastasi, M. Conti, M. D. Francesco, and A. Passarella, "Energy conservation in wireless sensor networks: A survey," Ad Hoc Networks, vol. 7, no. 3, pp. 537 - 568, 2009.

[8] Y. Yang, M. I. Fonoage, and M. Cardei, "Improving network lifetime with mobile wireless sensor networks," Computer Communications, vol. 33, pp. 409-419, March 2010.

[9] L. Ben-Saad and B. Tourancheau, "Towards an Optimal Positioning of Multiple Mobile Sinks in WSNs for Buildings," International Journal On Advances in Intelligent Systems IARIA, vol. 2, no. 4, pp. 411-421, 2009.

[10] L. Ben Saad and B. Tourancheau, "Sinks Mobility Strategy in IPv6based WSNs for Network Lifetime Improvement," in IFIP International Conference on New Technologies, Mobility and Security NTMS, 2011.

[11] T. Srisooksai, K. Keamarungsi, P. Lamsrichan, and K. Araki, "Practical data compression in wireless sensor networks: A survey," Journal of Network and Computer Applications, March 2011.

[12] A. T. Hoang and M. Motani, "Collaborative broadcasting and compression in cluster-based wireless sensor networks," ACM Transaction on Sensor Networks, vol. 3, August 2007.

[13] S. Agnihotri, P. Nuggehalli, and H. S. Jamadagni, "On maximizing lifetime of a sensor cluster," in Proceedings of the Sixth IEEE International Symposium on World of Wireless Mobile and Multimedia Networks, ser WOWMOM '05. Washington, DC, USA: IEEE Computer Society, 2005, pp. 312-317.

[14] P. Wang, C. Li, and J. Zheng, "Distributed data aggregation using clustered slepian-wolf coding in wireless sensor networks," in IEEE International Conference on Communications ICC, june 2007, pp. 3616 -3622 .

[15] F. Oldewurtel, J. Riihijärvi, and P. Mühönen, "Impact of correlation in node locations on the performance of distributed compression," in Proceedings of the Sixth international conference on Wireless OnDemand Network Systems and Services, ser. WONS'09. Piscataway, NJ, USA: IEEE Press, 2009, pp. 125-132.

[16] V. Jacobson, "TCP/IP compression for low-speed serial links," IETF, Tech. Rep. RFC 1144, 1990.

[17] M. Degermark, B. Nordgren, and S. Pink, "IP Header Compression," IETF, Tech. Rep. RFC 2507, 1999.

[18] L.-E. Jonsson, K. Sandlund, K. Sandlund, G. Pelletier, and P. Kremer, "RObust Header Compression (ROHC): Corrections and Clarifications to RFC 3095,” IETF, Tech. Rep. RFC 4815, 2007.
[19] G. Montenegro, N. Kushalnagar, J. Hui, and D. Culler, "Transmission of IPv6 Packets over IEEE 802.15.4 Networks," IETF, Tech. Rep. RFC 4944, September 2007.

[20] J. Hui and D. Culler, "Stateless IPv6 Header Compression for Globally Routable Packets in 6LoWPAN Subnetworks ," IETF, Tech. Rep. draftietf-6lowpan-hc-15, 2007.

[21] J. Hui and P. Thubert, "Compression Format for IPv6 Datagrams in 6LoWPAN Networks," IETF, Tech. Rep. draft-ietf-6lowpan-hc-13, September 2010.

[22] F. Kronewitter, "Dynamic Huffman addressing in wireless sensor networks based on the energy map," in IEEE Military Communications Conference MILCOM, 2008, pp. 1-6.

[23] W. R. Heinzelman, A. Chandrakasan, and H. Balakrishnan, "Energyefficient communication protocol for wireless microsensor networks," in Proceedings of the 33rd Hawaii International Conference on System Sciences, ser. HICSS '00. IEEE Computer Society, 2000, pp. 8020-.

[24] W. B. Heinzelman, A. P. Chandrakasan, and H. Balakrishnan, "An application-specific protocol architecture for wireless microsensor networks," IEEE Transactions on Wireless Communications, vol. 1, pp. 660-670, 2002

[25] D. Slepian and J. K. Wolf, "Noiseless coding of correlated information sources," IEEE Transactions on Information Theory, vol. 19, no. 4, pp. 471-480, July 1973.

[26] S. S. Pradhan and K. Ramchandran, "Distributed source coding using syndromes (DISCUS): design and construction," IEEE Transactions on Information Theory, vol. 49, no. 3, pp. 626-643, Mars 2003.

[27] A. Wyner, "Recent Results in the Shannon Theory," IEEE Transactions on Information Theory, vol. 20, no. 1, pp. 2-10, Mars 1974.

[28] L. Ben Saad, C. Lauradoux, B. Tourancheau, and F. Valois, "On the application of source-coding to addresses transmission," submitted to IEEE communication letters, 2011.

[29] F. MacWilliams and N. Sloane, The Theory of Error-Correcting Codes, twelth impression ed. North-Holland, 2006.

[30] M. Bhardwaj and A. Chandrakasan, "Bounding the lifetime of sensor networks via optimal role assignments," in INFOCOM 2002. TwentyFirst Annual Joint Conference of the IEEE Computer and Communications Societies. Proceedings. IEEE, vol. 3, 2002, pp. 1587 - 1596 vol.3. 